\section{Biosulfidogenesis in AMD: investigating the relation between the establishment of sulfidogenic microbial taxa and physicochemical conditions}

CHARLOTTE M VAN DER GRAAF ${ }^{1}$, ANDREY ILIN ${ }^{2}$, IÑAKI YUSTA ${ }^{2}$, FONS J.M. STAMS ${ }^{1,3}$, JAVIER SÁNCHEZESPAÑA $^{4}$ AND IRENE SÁNCHEZ-ANDREA ${ }^{1}$

${ }^{1}$ Wageningen University and Research

${ }^{2}$ University of the Basque Country

${ }^{3}$ University of Minho

${ }^{4}$ IGME

Presenting Author: lot.vandergraaf@wur.nl

Biogeochemical processes in anoxic water layers and sediments of areas affected by acid mine drainage (AMD) are strongly influenced by the activity of sulfidogenic microorganisms, such as sulfate-reducing bacteria (SRB). Biosulfidogenesis attenuates metal concentrations by enabling their removal, mainly through metal sulfides neoformation. In the case of SRB, sulfidogenesis at low $\mathrm{pH}$ furthermore has an alkalinizing effect due to the consumption of protons. However, the environmental factors that determine the establishment of sulfidogenic microorganisms, and how different sulfidogenic taxa correlate with physicochemical conditions are not yet clear.

We recently reported the natural attenuation of acidity and metal concentrations mediated by biosulfidogenesis in the water column of three acidic pit lakes (APL) in Spain: the oligotrophic pit lakes Filón Centro (FC) and La Zarza (LZ), and the eutrophic pit lake Brunita (BR). The putative SRB Desulfomonile accounted for $58.5 \%$ and $12.3 \%$ of reads in the monimolimnion of FC and BR, respectively, whereas in the more acidic LZ, the elemental sulfur $\left(\mathrm{S}^{0}\right)$-reducing genera Acidianus and Thermoplasma were detected. Based on our results, we hypothesized that biosulfidogenesis started with microbial $\mathrm{S}^{0}$ reduction, followed by sulfate reduction by SRB, and that establishment of SRB correlated with $\mathrm{pH}$. We further hypothesized that trophic (oligotrophic and eutrophic) state would correlate with the establishment of different SRB taxa.

We investigate these hypotheses using Winogradsky-type incubation columns (IC), inoculated with water and sediment from the mixolimnion and monimolimnion of $\mathrm{FC}$ and $\mathrm{BR}$. IC were incubated at room temperature in the dark for 18 months, with and without supplementation of organic carbon (glycerol). An unamended control column was incubated at $4^{\circ} \mathrm{C}$. By comparing the microbial community composition in the different IC at the end of incubation through 16S rRNA gene amplicon sequencing, we will investigate how specific sulfidogenic microbial taxa correlate with $\mathrm{pH}$, organic carbon availability, and nutrient content. The results will provide a better understanding of factors influencing biosulfidogenesis in AMD-affected environments and the role of biosulfidogenesis in element (e.g. $\mathrm{S}, \mathrm{Fe}, \mathrm{Cu}, \mathrm{Zn}, \mathrm{As}$ ) cycling in such extreme settings, and will contribute to the improvement of both bioremediation and 